\title{
Relationship between tremor and the onset of rapid voluntary contraction in Parkinson's disease
}

\author{
M M Wierzbicka, G Staude, W Wolf, R Dengler
}

Spinal Cord Injury Service, Brockton/ West Roxbury, VA Medical Center and Department of Neurology, Harvard Medical School Boston, MA 02132, USA M M Wierzbicka

Institute of Mathematics and Computer Science, Bundeswehr University, Munich, D-8014 Neubiberg, Germany G Staude W Wolf

Department of Neurology, University of Bonn, 5300 Bonn, Germany $R$ Dengler

Correspondence to: Dr M M Wierzbicka, Spinal Cord Injury Service, VA Medical Center, 1400 VFW Parkway, Boston, MA 02132, USA.

Received 11 March 1992 and in final revised form 15 September 1992

Accepted 28 September 1992

\begin{abstract}
It has been suggested that initiation of voluntary movement in Parkinson's disease (PD) is synchronous with tremor beats. This hypothesis was tested by examining quantitatively the onset of voluntary contraction in relation to the tremor cycle. Five PD patients with tremor at rest produced fast isometric abduction of the index finger in reaction time and selfpaced trials. For each trial, the time interval from last tremor peak to contraction onset was evaluated and its frequency distribution was compared with proposed statistical models. It was found that patients most often initiated contractions during the descending phase of the tremor oscillation and that the phasic EMG burst most commonly occurred after the mid-point of the EMG tremor cycle in both selfpaced and reaction time conditions. These results indicate that voluntary contraction is not initiated arbitrarily with respect to the tremor oscillation. Rather, there is a systematic phase relationship between the onset of the voluntary response and tremor. Thus attraction of voluntary movement to the tremor oscillator in PD results from abnormally synchronised motor units and failure to activate the motor neuron pool voluntarily soon after the tremor discharge.
\end{abstract}

$(\Im$ Neurol Neurosurg Psychiatry 1993;56:782-787)

Tremor is common in Parkinson's disease (PD). It is classified into different categories and named according to the condition that precipitates it as in resting tremor, action tremor, postural tremor, etc. Although clinically obvious tremor might not be seen under one condition it can appear, even in akinetic patients, ${ }^{1}$ when the muscle state is voluntarily changed-for example, from rest to active contraction. Because tremor is often present during performance of voluntary motor actions it could contribute to the impairment of voluntary motor behaviour in PD. Some observations have already been made on the possible interaction between tremor and voluntary movement. Hallett $e t a^{2}$ suggested that "some delay in initiating movement in patients with tremor at rest might come from 'waiting to get into correct time of the cycle' ". Studies of repetitive finger tapping movements showed that at certain cue frequencies approaching tremor frequencies, PD patients produce movements faster (hastening phenomenon) or slower than intended. ${ }^{34}$ Since hastened voluntary responses were performed at tremor frequencies, this effect was assumed $^{3}$ to be caused by an attraction of voluntary movements to the tremor oscillator.

To provide direct evidence that tremor has an effect on the voluntary motor response, the relationship between tremor and the onset of the response has to be examined quantitatively. The purpose of this study was to investigate the phase relationship between isometric resting tremor and the onset of the rapid voluntary contraction to determine whether the initiation of a voluntary response is arbitrary with respect to tremor, or occurs during a certain phase of the tremor cycle.

\section{Methods}

DATA ACQUISITION

We studied five patients, aged 55-76 years, with tremor at rest. All patients followed their normal schedule of medication and experiments were performed just before the next dosage. During the experiment the patient was seated comfortably in a chair with hand and forearm supported on a table and strapped to a heavy metal plate. The index finger was immobilised in a metal rail which was attached to a bipolar pull-push force transducer (capacity $10 \mathrm{~kg}$ ). Actual contraction force was displayed on a colour video monitor in front of the subject as a vertically moving bar. At the beginning of the experiment, the subject was instructed to maintain this bar at a horizontal broken line indicating resting position and then to produce a contraction "as fast as possible" to a target shown with a solid line. These resting and target lines were displayed on the screen at all times during the experiment.

Patients performed fast isometric index finger abductions in both reaction time (RT) and selfpaced (SP) conditions. In RT trials the subject was asked to react "as soon as possible" when the screen background colour changed from black to red. There was no visual stimulus used in SP trials (screen stayed the same colour) and the only verbal instruction given to the subject was "respond when ready". After a short practice session, the patients produced 50-100 contractions for each condition. Force and electromyographical (EMG) signals were acquired for six seconds with $1 \mathrm{kHz}$ sampling rate. The EMG was recorded from the first dorsal 

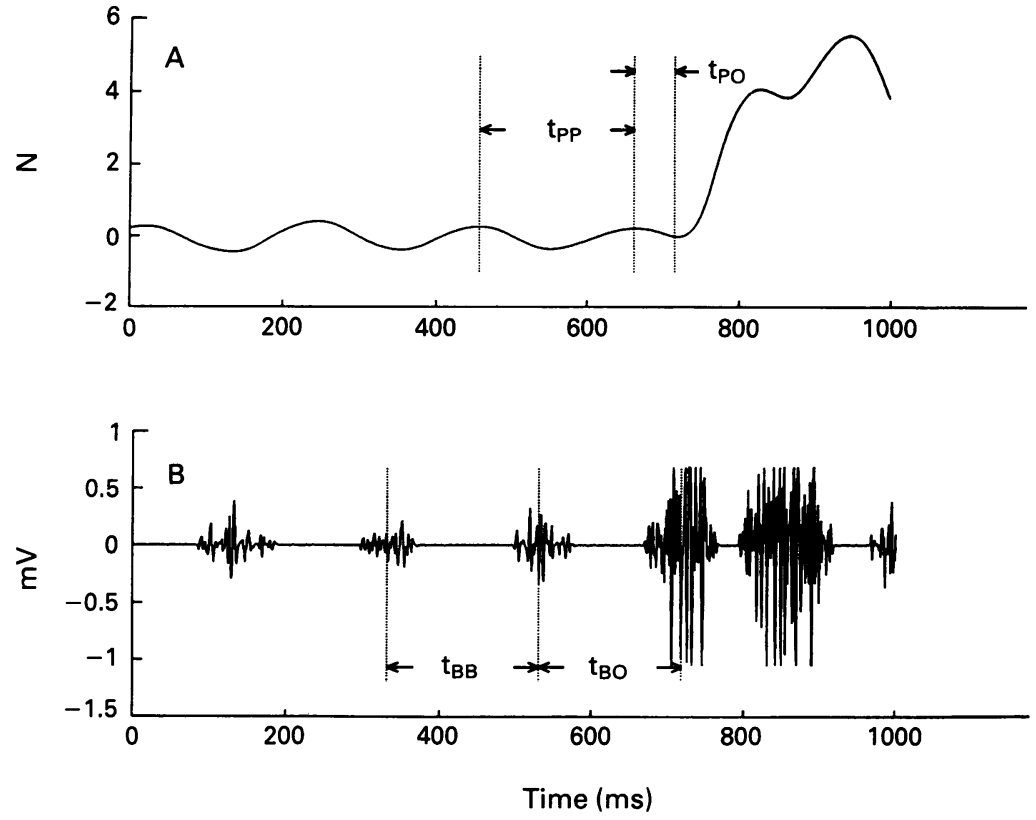

Figure 1 (A) Single trial of force recording. For each contraction two time intervals were calculated $t_{P P}$ peak to peak interval before voluntary contraction and $t_{P O}$, peak to onset interval; $(B)$ The corresponding EMG recording. The $t_{B B}$ interval between the gravity centres of the two last tremor bursts and the $t_{B O}$ interval from the last tremor burst to the first phasic burst were determined.

interosseous muscle (FDI) with surface Liberty Mutual electrodes (MYO-111, 3dB bandwidth of $120-500 \mathrm{~Hz}$ ). Data were analysed offline on a MicroVax II system.

\section{DATA EVALUATION}

The force signal was smoothed using a Gaussian low-pass filter with a bandwidth of $50 \mathrm{~Hz}$. For each contraction the time intervals between the last two tremor peaks before the voluntary contraction $\left(t_{\mathrm{PP}}=\right.$ peak to peak interval) and from the last tremor peak to the response onset $\left(t_{P O}=\right.$ peak to onset interval) were evaluated (fig 1A). For each corresponding $E M G$ recording, time intervals $t_{B B}$ between the gravity centres of the two last EMG tremor bursts and $t_{B O}$ from the last tremor burst to the first phasic burst were calculated (fig 1B). The $t_{P O}$ and $t_{B O}$ intervals were then normalised with respect to the length of tremor cycle and expressed in degrees to indicate phase relationship

$$
\begin{aligned}
& \varphi_{\mathrm{PO}}=\mathrm{t}_{\mathrm{PO}} / \mathrm{t}_{\mathrm{PP}} \times 360^{\circ} \\
& \varphi_{\mathrm{BO}}=\mathrm{t}_{\mathrm{BO}} / \mathrm{t}_{\mathrm{BB}} \times 360^{\circ}
\end{aligned}
$$

where $360^{\circ}$ corresponds to the full length of the tremor cycle.

The contraction onset was detected with an automatic routine which is described in the Appendix. The effectiveness of the onset detection algorithm was tested on computer simulated force trials. The discrepancies between distributions of real and detected onset are discussed in the Appendix. In general, error of the onset detection in simulated trials was very small. For five tested statistical distributions of the onset (see section below, models 1 to 5 ) the rate of the correct onset detections by the automatic routine was higher than $95 \%$. Nevertheless, in each experimental trial, automatic onset detection was additionally confirmed by visual inspection. More than $70 \%$ of all voluntary contractions were included in the final statistical evaluation. The reason for rejection of a trial was either lack of an evident resting tremor before voluntary contraction (about $10 \%$ ) or poor quality EMG recordings (about $20 \%$ ).

\section{MODELLED DISTRIBUTIONS}

In order to investigate the phase relationship between contraction initiation and resting tremor the measured frequency distribution of $\varphi_{\mathrm{PO}}$ phases was compared with five modelled distributions. Each modelled distribution was generated with computer simulated force data of 500 trials (Appendix). The statistical distribution of the onset phase for simulated data was different for each model. Model 1 postulated no relationship between tremor and the onset of voluntary contraction and was represented by a uniform distribution of the onset over the tremor cycle (fig 2A). Model 2 assumed the voluntary contraction to be initiated with equal probability over the entire descending phase of the tremor

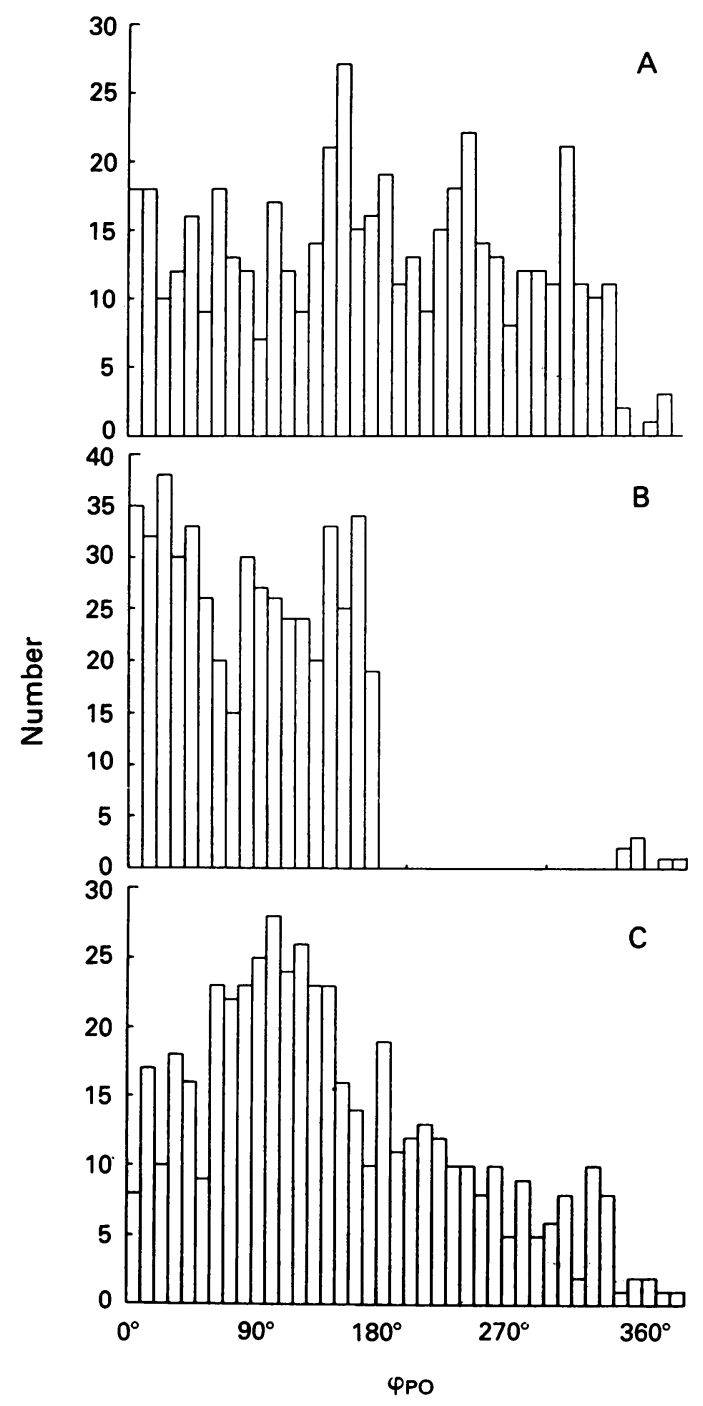

Figure 2 Onset phase distributions resulting from different statistical models. (A) model 1 postulates uniform distribution throughout the tremor cycle; (B) model 2 assumes uniform distribution on the descending phase of the tremor cycle; (C) model 3 represents Gaussian distribution on the descending phase of tremor centred at $120^{\circ}\left(S D 90^{\circ}\right)$. 
cycle and was represented by uniform distribution over the first half of the tremor cycle (fig 2B). Models 3, 4, and 5 proposed more exact phase relationships between the onset of voluntary contraction and tremor. Model 3 assumed a Gaussian distribution over the descending slope with mean of $120^{\circ}\left(\mathrm{SD} 90^{\circ}\right)$ (fig 2C), model 4 Gaussian distribution centred at the valley of the tremor cycle (mean $180^{\circ}, \mathrm{SD} 90^{\circ}$ ), and model 5 Gaussian distribution on the ascending slope (mean $270^{\circ}$, $\operatorname{SD} 90^{\circ}$ ).

\section{Results}

The force tremor frequency (reciprocal of median $t_{P P}$ interval) for combined SP and RT data ranged in patients from 2.4 to $5.3 \mathrm{~Hz}$ and almost identical values were obtained with EMG measurements (reciprocal of median $t_{B B}$ interval, table 1 ). We found no significant differences in the initiation of the voluntary contraction in relation to the tremor cycle among patients and in between SP and RT trials within each patient (table 2). In addition, the statistical analysis of the EMG data showed that the phasic EMG burst was timed in relation to the EMG tremor cycle in a similar way in all patients under both SP and RT conditions (except patient 4 in whom SP/RT difference reached borderline significance (table 2). The $\varphi_{\mathrm{PO}}$ median values tended to fall (table 2) into the second quarter of the tremor cycle $\left(90^{\circ}-180^{\circ}\right)$ and median values of $\varphi_{\mathrm{BO}}$ into the last quarter $\left(270^{\circ}-360^{\circ}\right)$. In general, $70 \%$ of the 562 voluntary contractions that were analysed were initiated on the descending slope during the first half of the peak to peak tremor cycle $\left(0^{\circ}-180^{\circ}\right)$. In $90 \%$ of trials, the phasic voluntary EMG burst was generated after the mid-point of the EMG tremor cycle

Table 1 Median values and $95 \%$ confidence intervals of $E M G$ and the force tremor cycle length for combined SP and $R T$ data

\begin{tabular}{lll}
\hline $\begin{array}{l}\text { Patient } \\
\text { No }\end{array}$ & $\begin{array}{l}\text { EMG interval } \\
t_{B B}(m s)\end{array}$ & $\begin{array}{l}\text { Force interval } \\
t_{P P}(m s)\end{array}$ \\
\hline 1 & $188\left[\begin{array}{lll}183 & 194\end{array}\right]$ & $188\left[\begin{array}{ll}184 & 191\end{array}\right]$ \\
2 & $191\left[\begin{array}{ll}188 & 195\end{array}\right]$ & $190\left[\begin{array}{ll}186 & 193\end{array}\right]$ \\
3 & $213[209218]$ & $210[204217]$ \\
4 & $268\left[\begin{array}{ll}256 & 275\end{array}\right]$ & $263\left[\begin{array}{ll}253 & 270\end{array}\right]$ \\
5 & $411\left[\begin{array}{ll}380 & 433\end{array}\right]$ & $409\left[\begin{array}{ll}377 & 430\end{array}\right]$ \\
Mean & $217\left[\begin{array}{ll}211 & 222\end{array}\right]$ & $217\left[\begin{array}{ll}210222\end{array}\right]$ \\
\hline
\end{tabular}

Table 2 Median values, $95 \%$ confidence intervals and results of non-parametric tests for comparison between experimental conditions (SP vs RT) for each patient (U test, Wilcoxon-Mann-Whitney ${ }^{21}$ ) and across patients ( $H$ test of Kruskal-Wallis ${ }^{21}$ ).

\begin{tabular}{|c|c|c|c|c|c|c|}
\hline \multirow{2}{*}{$\begin{array}{l}\text { Patient } \\
\text { No }\end{array}$} & \multicolumn{2}{|c|}{$E M G$ phase $\varphi_{B O}($ deg $)$} & \multirow{2}{*}{$\begin{array}{c}\text { Difference } \\
-S P / R T \\
\text { U test }\end{array}$} & \multicolumn{2}{|c|}{ Force phase $\varphi_{B O}$ (deg) } & \multirow{2}{*}{$\begin{array}{l}\text { Differences } \\
S P / R T \\
U \text { test }\end{array}$} \\
\hline & $S P$ & $R T$ & & $S P$ & $R T$ & \\
\hline $\begin{array}{l}1 \\
2 \\
3 \\
4 \\
5\end{array}$ & $\begin{array}{l}324\left[\begin{array}{ll}294 & 348\end{array}\right] \\
348\left[\begin{array}{ll}292 & 362\end{array}\right] \\
273\left[\begin{array}{ll}248 & 344\end{array}\right] \\
282\left[\begin{array}{lll}238 & 307\end{array}\right] \\
297\left[\begin{array}{ll}236 & 329\end{array}\right]\end{array}$ & $\begin{array}{l}300\left[\begin{array}{ll}280 & 313\end{array}\right] \\
320\left[\begin{array}{ll}290 & 341\end{array}\right] \\
281\left[\begin{array}{lll}271 & 327\end{array}\right] \\
313\left[\begin{array}{ll}295 & 337\end{array}\right] \\
304\left[\begin{array}{lll}233 & 337\end{array}\right]\end{array}$ & $\begin{array}{l}1 \cdot 31 \\
1 \cdot 29 \\
0 \cdot 78 \\
2 \cdot 18^{\star} \\
0.04\end{array}$ & $\begin{array}{l}125\left[\begin{array}{ll}103 & 176\end{array}\right] \\
171\left[\begin{array}{lll}144 & 196\end{array}\right] \\
143\left[\begin{array}{lll}116 & 187\end{array}\right] \\
172\left[\begin{array}{lll}121 & 208\end{array}\right] \\
130\left[\begin{array}{ll}79 & 183\end{array}\right]\end{array}$ & $\begin{array}{l}149\left[\begin{array}{ll}107 & 175\end{array}\right] \\
141[114172] \\
202[192207] \\
179\left[\begin{array}{ll}142 & 215\end{array}\right] \\
148[108178]\end{array}$ & $\begin{array}{l}0.32 \\
1.37 \\
0.64 \\
0.41 \\
0.82\end{array}$ \\
\hline$H$ test & 8.97 & \multicolumn{5}{|c|}{$\begin{array}{l}\text { Differences between patients for SP and RT trials } \\
4.26 \\
3.08\end{array}$} \\
\hline
\end{tabular}

$\left(180^{\circ}-360^{\circ}\right)$. The difference in percentages of the EMG and force evaluation is produced by the onset detection procedure which biases both $\varphi_{\mathrm{PO}}$ and $\varphi_{\mathrm{BO}}$ distributions but in different ways (Appendix).

The frequency distributions of $\varphi_{\mathrm{PO}}$ and $\varphi_{\mathrm{BO}}$ phases for all data combined are shown in figure 3. It is noteworthy that the force distribution $\left(\varphi_{\mathrm{PO}}\right)$ is right-skewed. The asymmetry in force distribution is seen (fig 3A) because the contraction onset occurs at an early part of the tremor cycle. Therefore onsets which would belong to the left hypothetical tail of the distribution (negative onset phase $\varphi_{\mathrm{PO}}$ ) are referred to one tremor peak earlier and appear in the right tail of the distribution (see Appendix). For example, the onset occurrence at $-100^{\circ}$ is transformed to $260^{\circ}$. The force was also analysed in the trials rejected because EMG recordings were not of adequate quality. However, these results were not included in the final statistics for the sake of consistency in data presentation, thus allowing for direct comparison of force and EMG distributions (fig 3). In general, the distribution of contraction onset phase in these rejected trials was similar to that shown in figure $3 \mathrm{~A}$.

The most important information contained in both the EMG and force distributions is a distinct preference to initiate voluntary contraction during certain phases of the tremor cycle. As can be seen in the EMG distribution (fig $3 \mathrm{~B}$ ) there is a very small probability of the occurrence of the phasic EMG burst before the mid-point of EMG tremor cycle. A comparison of the empirical $\varphi_{\mathrm{PO}}$ distribution (fig 3A) with modelled distributions shows that only the distribution proposed with Model 3 (fig 2C) fits the experimental data; all other distributions differ significantly (table 3). Model 3 assumes that the onset of voluntary contraction has a Gaussian distribution over the tremor cycle with mean value of $120^{\circ}\left(\mathrm{SD} 90^{\circ}\right)$. This result implies that there is a systematic phase relationship between the onset of voluntary contraction and tremor.

\section{Discussion}

Our data indicate that the initiation of voluntary contraction tends to occur during the descending phase of the tremor cycle. Moreover, the onset is not uniformly distributed during this phase (as proposed by model 2). Rather, there is a systematic phase relationship between tremor and the onset of voluntary contraction as in model 3, which provides a good fit to the experimental data. The phasic EMG burst is most commonly generated during the second half of the EMG tremor cycle. It occurs slightly earlier (most often at $300^{\circ}$ ) than expected from the resting tremor burst $\left(360^{\circ}\right.$ fig $\left.3 \mathrm{~b}\right)$. The shorter interburst interval might reflect the transition from resting to faster action tremor.

Current data provide quantitative evidence that the initiation of a voluntary motor response is attracted to the tremor oscillator 

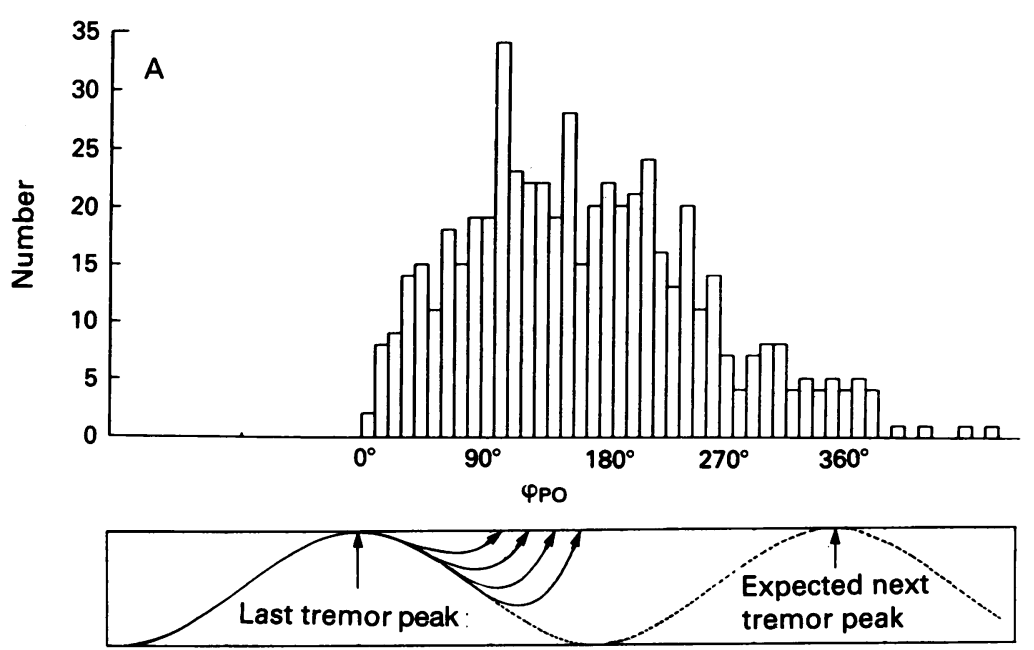

Onset of contraction
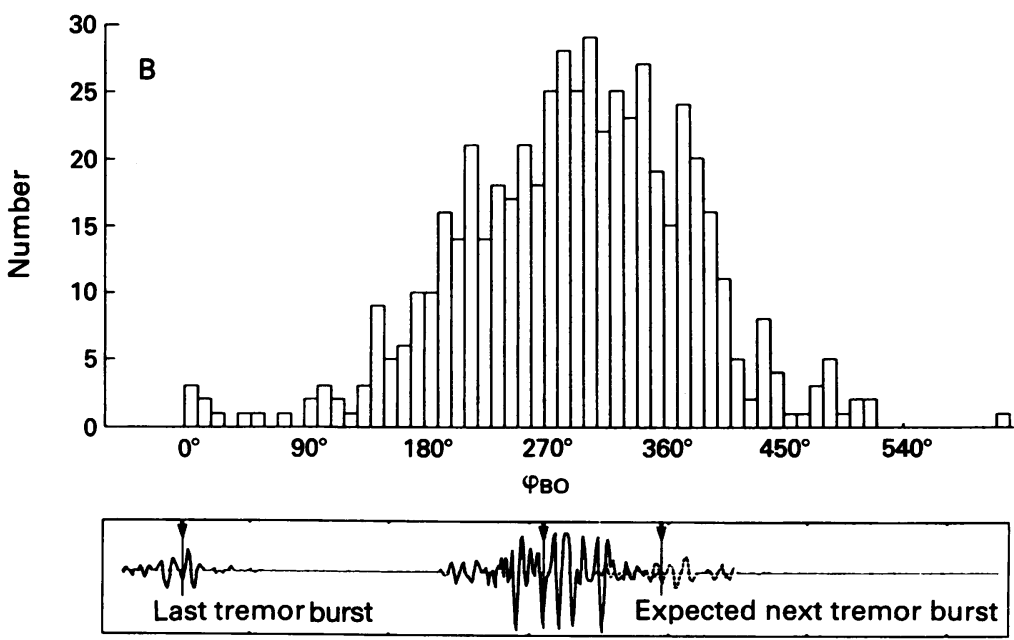

First phasic burst

Figure 3 Frequency distribution of: $(A) \varphi_{P O}$ phase between the last force tremor peak and contraction onset; (B) $\varphi_{B O}$ phase between the last tremor EMG burst and the first phasic EMG burst; obtained from evaluation of 562 voluntary contractions. Inserts below provide an illustration of force and EMG tremor cycles.

in PD. This finding confirms the observation of Hallet et $a l^{2}$ that single rapid voluntary movements are timed in relation to tremor. The existence of a systematic phase relationship between tremor and voluntary response may account for the observation of Logigian et $a l^{3}$ that hastening or slowing of paced voluntary alternating motor responses are forced to tremor frequencies (basic frequency and its subharmonics). An attraction of voluntary movement onset to tremor was also reported for normal subjects 5 even though physiological tremor is much weaker than Parkinsonian pathological tremor. Goodman and Kelso ${ }^{5}$ found that ballistic movements are initiated

Table 3 Comparison between the empirical distribution of the contraction onset phase (fig $3 a$ ) and modelled distributions ( $\chi^{2}$ goodness of fit test).

\begin{tabular}{llc}
\hline Model & Distribution & $\chi^{2}$ \\
\hline 1 & uniform, whole interval $0-360^{\circ}$ & $141 \cdot 9^{\star}$ \\
2 & uniform, descending phase $0-180^{\circ}$ & $433 \cdot 0^{\star}$ \\
3 & normal, descending phase $120^{\circ}\left(90^{\circ}\right)$ & $24 \cdot 4$ \\
4 & normal, valley $180^{\circ}\left(90^{\circ}\right)$ & $69 \cdot 3^{\star}$ \\
5 & normal, ascending phase $270^{\circ}\left(90^{\circ}\right)$ & $280 \cdot 7^{\star}$ \\
\hline ¿Significant difference $\left(\chi^{2}=25\right.$ is the critical value for signifi- \\
cance at 5\% level, ${ }^{21} 15$ degrees of freedom, range $\left.0-450^{\circ}\right)$.
\end{tabular}

around the point of maximum angular velocity (at the ascending phase of tremor oscillation) which is mechanically the most energy efficient time to start the movement. Although we observed the voluntary response onset to be during the descending rather than the ascending phase of the tremor cycle, it is difficult to compare our results directly to those of Goodman and Kelso ${ }^{5}$ because of differences in the paradigm used (isometric vs isotonic) and in the nature of the tremor being investigated (pathological vs physiological).

It could be argued that changes in muscle length or Ia discharge from muscle spindles might play a role in enhancing onset of the voluntary contraction during the descending phase of tremor oscillation. Since these experiments were performed in an isometric condition, variation in muscle length was small and should not have a significant effect on force generated during voluntary contraction. It is also unlikely that discharge from muscle spindles would increase probability to start the voluntary contraction at the descending slope. Microneurographic recordings from finger flexors (isometric condition) during tremor at rest in patients with $P D$ showed that, most often, firing of spindles occurred during both the relaxation and contraction phases, and rarely during the relaxation phase alone. ${ }^{6}$

We suggest that attraction to the tremor oscillator in PD results from the discharge of abnormally synchronised motor units and failure to activate the motor neuron pool voluntarily soon after the tremor discharge. Slower firing rates and, in general, prolonged post-excitatory inhibition between synchronous discharges have been previously reported in PD patients. ${ }^{7-9}$ Since an attraction phenomenon can produce some delays in response initiation it may account for prolonged intermovement times in movement sequences $^{1011}$ and also for the slow rise in force in PD. ${ }^{12-16}$ In rapid voluntary motor responses of bradykinetic $P D$ patients the first EMG burst is often of insufficient magnitude and additional EMG bursts are required to accomplish the task. ${ }^{121617}$ Thus to initiate each consecutive contraction the necessary "waiting" time (presumably shorter when larger forces are reached because action tremor is faster than resting tremor) may explain lack of smoothness and force irregularities, such as characteristic staircase profiles, observed in both slow $^{15}$ and fast contractions. ${ }^{16}$

Since, in general, PD patients underestimate the muscle activity required for a task, Berardelli et al ${ }^{12}$ suggested that in PD the link between "perceptual appreciation" of the task and "delivery of appropriate instructions" to the motor cortex is broken. However, other studies showed that PD patients have an adequate internal model of the forces required to produce movements ${ }^{15}$ and that the overall motor programme seems to be intact. ${ }^{16} 18-20$ The present study supports the view that abnormal voluntary motor behaviour in $\mathrm{PD}$ 
Figure A1 Automatic onset detection procedure. The force signal, and its first and second derivatives are shown. The tolerance window around each waveform is marked with broken lines. The earliest departure of one of these signals from the window was defined as a contraction onset.
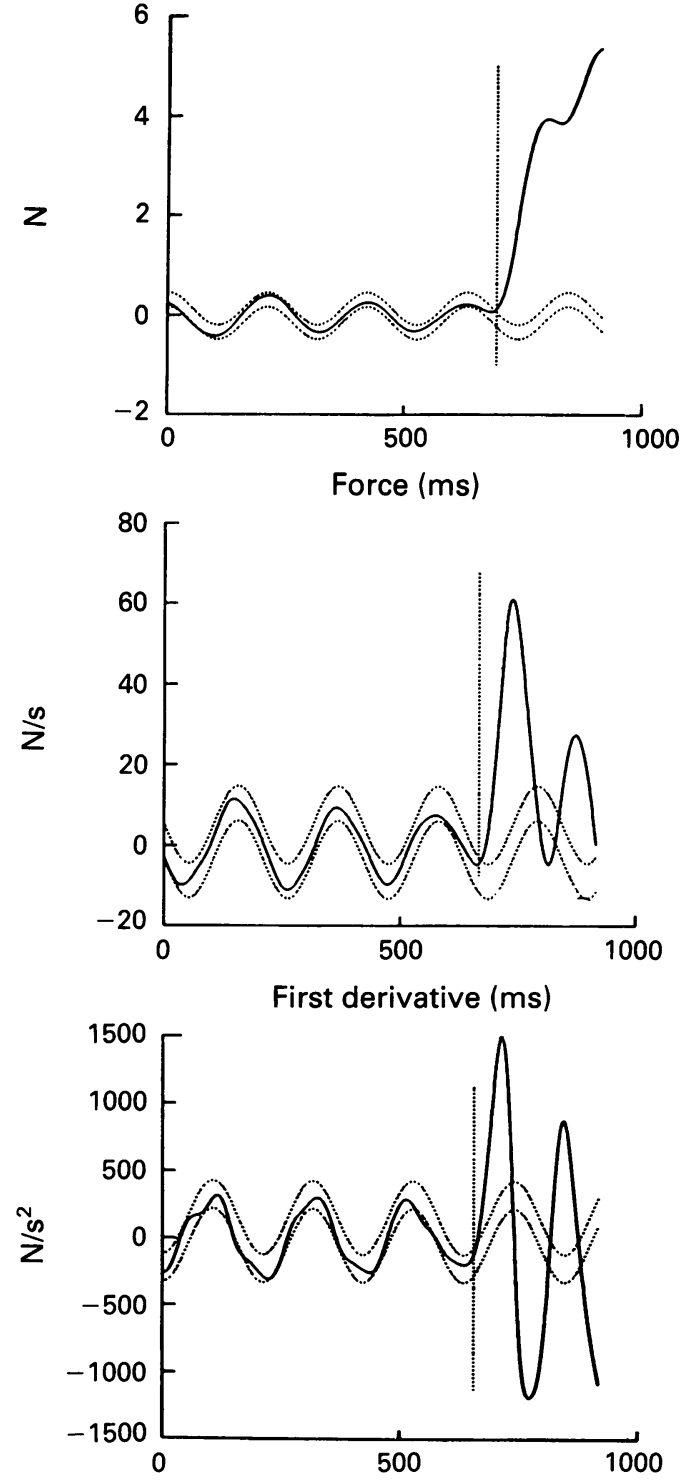

Second derivative (ms)

\section{Appendix}

THE AUTOMATIC ONSET DETECTION PROCEDURE

The contraction onset searching algorithm was based on detection of the departure of force and its higher derivatives from the extrapolated tremor waveform (fig A1). The force tremor signal was approximated by a third order polynomial with a superimposed sinewave as follows:

$$
\begin{gathered}
y(t)=a_{3} t^{3}+a_{2} t^{2}+a_{1} t+ \\
a_{0}+A \cos \left(2 \pi t / t_{p P}+d\right)
\end{gathered}
$$

For each experimental trial the coefficients $\left(a_{i}, A, d\right)$ were determined so as to minimise mean square error between the measured and modelled force tremor (1) within a time interval of three tremor cycles before the voluntary contraction. The onset of contraction was considered to occur when the signal exceeds a window of certain width ( \pm maximum error during last three tremor periods, fig A1). To increase the accuracy of onset detection (in particular during ascending tremor phase) the same procedure was applied to the first and second force derivatives. The earliest of three detected onsets (force $\mathrm{F}, \mathrm{dF} / \mathrm{dt}, \mathrm{d}^{2} \mathrm{~F} / \mathrm{dt}^{2}$ was defined to be the onset of the voluntary contraction.

TEST OF THE ONSET DETECTION PROCEDURE ON COMPUTER SIMULATED DATA

In a simulated force trial, the tremor was represented by a sinewave with variability in the length/amplitude parameters of the cycle similar to that observed in PD subjects (length $\mathrm{SD}=30 \mathrm{~ms}$, amplitude $\mathrm{SD}=0.2 \mathrm{~N}$ ) and a voluntary contraction was modelled by a cubic spline function (fig A2). For each tested statistical distribution of the contraction onset (models 1 to 5 ) there was always a strong correlation $(r>0.97)$ between the real onset (merging point between sinewave and spline function) and computer detected onset as indicated by the main diagonal cluster of points (fig A3). However, in addition to the main cluster, two smaller clusters of points

might be related to a deficit in control of motor unit firing. ${ }^{1516}$ This deficit includes failure to produce phasic EMG bursts of sufficient magnitude ${ }^{1617}$ and, as shown in the present study, inability to initiate voluntary muscle activity in a continuous way.

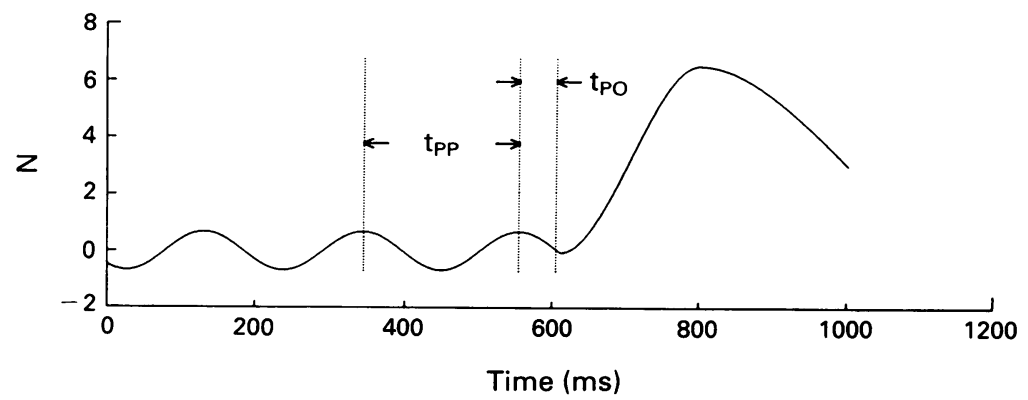

Figure A2 Simulated force trial. Tremor is modelled with a sinewave and voluntary contraction with a cubic spline function.

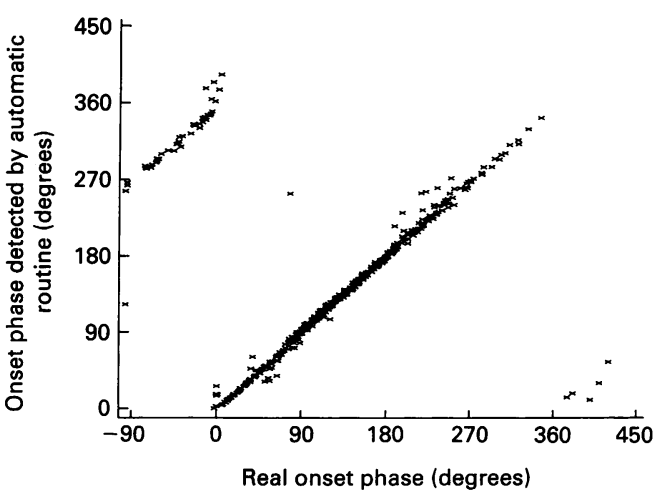

Figure A3 Test of the onset detection procedure performed on 500 simulated force trials with onset having Gaussian distribution at $120^{\circ}\left(S D 90^{\circ}\right)$. The relationship between real (merging point between sinewave and spline function) and detected onset is shown. Note a strong correlation between both onsets indicated by the main diagonal cluster of points. Small corner components are consequences of the methodology used for onset evaluation in periodic data (Appendix). 
were also formed (fig A3). To explain the upper left cluster, assume the real onset has a Gaussian distribution centred at $120^{\circ}$ on the descending tremor slope with $\mathrm{SD}=90^{\circ}$, as proposed in model 3. The onsets which happen at a distance larger than $120^{\circ}$ to the left of centre of the distribution would occur before the last tremor peak. This left tail of Gaussian distribution (negative phase, $\varphi_{\mathrm{PO}}<$ 0 ) is detected by the applied procedure at $360^{\circ}+\varphi_{\mathrm{PO}}$ because it is referred to one tremor peak earlier (which in this case is the last tremor peak before contraction, fig A3).

Thus our methodology for onset evaluation transforms the original symmetric Gaussian distribution into an asymmetric distribution. The amount of symmetry distortion depends on the position of the centre of the distribution in relation to the tremor cycle. The low right cluster of points in fig A3 can be explained by random fluctuations in the length of the tremor cycle. It is then statistically possible that in some trials the length of the last tremor cycle $\left(t_{\mathrm{PP}}\right)$ can be shorter than peak to onset interval $\left(t_{P O}\right)$. In such cases the onset is detected at phases greater than $360^{\circ}$ (fig A3). It is worth emphasising that neither of these effects contributes to error in the evaluation of onset but they do indicate differences between real and detected onset distributions which are consequences of the methodology used to evaluate periodic data with random variability of the cycle. This fact has to be taken into account in order to compare modelled distributions with those obtained from experiments. Thus modelled distributions shown in fig 2 represent frequency distributions of the automatically detected onset in simulated force data in which real onset had statistical distributions as postulated with models 1 to 3 .

These effects are also present in the EMG evaluation. However, the results of force and EMG analysis are biased in different ways because there is a phase difference between EMG and force as well as in the location of the onset in relation to the tremor cycle. Therefore onset evaluation based on EMG and force can differ somewhat, as can be seen in the results section.
The authors are grateful to Drs WH Oertel and J Schwarz from the Department of Neurology, University of Munich for providing access to the patients and to Drs EL Logigian and AW Wiegner for their useful comments on the manuscript.
This study was supported by a grant from Deutsche This study was supported by
Forschungsgemeinschaft (DFG).

1 Teravainen H, Calne DB. Action tremor in Parkinson's disease. F Neurol Neurosurg Psychiatry 1980;43:257-63.

disease. $\mathcal{F}$ Neurol Neurosurg Psychiatry 1980;43:257-63.
2 Hallett M, Shahani BT, Young RR. Analysis of stereotyped voluntary movements at the elbow in patients with Parkinson's disease. $\mathcal{F}$ Neurol Neurosurg Psychiatry 1977;40:1129-35.

3 Logigian E, Hefter H, Reiners K, Freund H-J. Does tremor pace repetitive voluntary motor behavior in Parkinson's disease? Ann Neurol 1991;30:172-9.

4 Nakamura R, Nagasaki H, Narabayashi H. Disturbances of rhythm formation in patients with Parkinson's disease: part I. Characteristics of tapping response to the periodic signals. Percept Mot Skills 1978;46:63-75.

5 Goodman D, Kelso JAS. Exploring functional significance of physiological tremor: a biospectroscopic approach. Exp Brain Res 1983;49:419-31.

6 Hagbarth KE, Wallin G, Löfstedt L, Aquilonius SM. Muscle spindle activity in alternating tremor of Parkinsonism and in clonus. $f$ Neurol Neurosurg Psychiatry 1975;38:636-41.

7 Dengler R, Wolf $\mathbb{W}$, Schubert M, Struppler A. Discharge pattern of single motor units in basal ganglia disorders. Neurology 1986;36:1061-6.

8 Dietz V, Hillesheimer W, Freund H-J. Correlation between tremor, voluntary contraction, and firing pattern of motor units in Parkinson's disease. $\mathcal{F}$ Neurol Neurosurg Psychiatry 1974;37:927-37.

9 Milner-Brown HS, Fischer MA, Weiner J. Electrical properties of motor units in parkinsonism and a possible relationship with bradykinesia. $f$ Neurol Neurosurg Psychiatry 1979;42:35-41.

10 Benecke R, Rothwell JC, Dick JPR, et al. Disturbance of sequential movements in patients with Parkinson's disease. Brain 1987;110:361-79.

11 Stelmach GE, Worringham CJ, Strand EA. Programming and execution of movement sequences in Parkinson's disease. Int $\mathcal{F}$ Neurosci 1987;36:55-65.

12 Berardelli A, Dick JPR, Rothwell JC, et al. Scaling of the size of the first agonist EMG burst during rapid wrist movements in patients with Parkinson's disease. $\mathcal{f}$ Neurol Neurosurg Psychiatry 1986;49:1273-9.

13 Flowers KA. Visual 'closed-loop' and 'open-loop' characteristics of voluntary movements in patients with parkinsonism and intention tremor. Brain 1976;99:269-310.

14 Sheridan MR, Flowers KA, Huller J. Programming and execution of movement in Parkinson's disease. Brain 1987;110:1247-71.

15 Stelmach GE, Teasdale N, Phillips J, Worringham CJ. Force production characteristics in Parkinson's disease. Force production characteristics
Exp Brain Res 1989;76:165-72.

16 Wierzbicka MM, Wiegner AW, Logigian EL, Young RR. Abnormal most-rapid isometric contractions in patients with Parkinson's disease. $\mathcal{F}$ Neurol Neurosurg Psychiatry 1991;54:210-6.

17 Hallett $M$, Koshbin S. A physiological mechanism of bradykinesia. Brain 1980;115:465-80.

18 Bloxham CA, Mindel TA, Frith CD. Initiation and execution of predictable and unpredictable movements in Parkinson's disease. Brain 1984;107:371-84.

19 Evarts EV, Teravainen $H$, Calne DB. Reaction time in Parkinson's disease. Brain 1981;104:167-86.

20 Rafal RD, Inhoff AW, Friedman, JH Bernstein E. Programming and execution of sequential movements in Programming and execution of sequential movements in 1987;50:1267-73.

21 Sachs L. Applied statistics: a handbook of techniques, 2nd ed. New York: Springer Verlag, 1984. 